\title{
Epithelial Area Detection in Cytokeratin Microscopic Images Using MSER Segmentation in an Anisotropic Pyramid
}

\author{
Cristian Smochina ${ }^{1}$, Radu Rogojanu ${ }^{2}$, Vasile Manta ${ }^{1}$, and Walter Kropatsch ${ }^{3}$ \\ ${ }^{1}$ Faculty of Automatic Control and Computer Engineering, \\ "Gheorghe Asachi" Technical University of Iasi, Romania \\ smochina.cristian@yahoo.com, vmanta@cs.tuiasi.ro \\ ${ }^{2}$ Institute of Pathophysiology and Allergy Research, Medical University, Vienna, Austria \\ radu@rogojanu.com \\ ${ }^{3}$ Pattern Recognition and Image Processing Group, Institute of Computer Graphics and \\ Algorithms, Vienna University of Technology, Austria \\ krw@prip.tuwien.ac.at
}

\begin{abstract}
The objective of semantic segmentation in microscopic images is to extract the cellular, nuclear or tissue components. This problem is challenging due to the large variations of features of these components (size, shape, orientation or texture). In this paper we present an automatic technique to robustly delimit the epithelial area (crypts) in microscopic images taken from colon tissues sections marked with cytokeratin-8. The epithelial area is highlighted using the anisotropic diffusion pyramid and segmented using MSER+. The crypts separation and lumen detection is performed by imposing topological constraints about the epithelial layer distribution within the tissue and the round-like shape of the crypt. The evaluation of the proposed method is made by comparing the results with ground-truth segmentations.
\end{abstract}

Keywords: Crypt segmentation, Anisotropic diffusion pyramid, MSER, Biomedical imaging, Pathology, Microscopy, Fluorescence microscopy, Image analysis.

\section{Introduction}

In diagnostic pathology, the pathologists give a diagnostic after a set of biological samples (tissues stained with different markers) are viewed and many specific features of the objects of interest (size, shape, colour or texture) have been analysed. This time consuming and tedious process is an important part in clinical medicine but also in biomedical research and can be enhanced by providing the pathologists/biologists with quantitative data extracted from the images.

To overcome also the possible subjectivity caused by different visual interpretations of different pathologists, image processing techniques are used to allow large scale statistical evaluation in addition to classical eye screening evaluation. 
Image segmentation of microscopic images is a hard task due to several elements of variability noticed in the image dataset like size and arrangement of the crypts inside the tissue sections, intensity levels of both background and cells from either stroma or crypts, as well as a relatively high background compared to the objects of interest.

In this paper we focus on analysing the tissue components like crypts, lumen and stroma, without dealing directly with the small objects like nuclei and cells (Fig. 1a). A rough description and a short overview of the problems to be solved are presented bellow; each step of the proposed method will present more details and justifications:

- some images are slightly underexposed due to weak local biological response.

- some image portions don't contain useful information.

- the crypt appears like a "donut" (or 2D projection of a torus) with many small 'holes' and a central black area inside (lumen).

- the lumen is a black area with different sizes surrounded by the epithelial layer.

- the "donut" has a non-homogeneous organization due to the dark regions smaller than the lumen.

- the pixels within a crypt correspond to three main components: background, dark regions and the noisy pixels, weak biological binding response and strong response (highest values).

- the stroma separates the crypts; situations of touching/very close crypts can appear.

- no relevant information or cells exist between touching/very close crypts.

- the number of crypts may be used in computing distribution statistics.

This paper is organized as follows. The last part of this section points out the goal of this study. The images are enhanced in Section 2 and the epithelial area is highlighted using the anisotropic diffusion pyramid (Section 3.1) and segmented by MSER+ (Section 3.2). In Section 4.1 the false positive results are reanalysed for crypts separation and the lumens are detected in 4.2. The results are evaluated and discussed in Section 5 while the conclusions are elaborated in Section 6.

\subsection{State of the Art}

The detection of histological structures like gland or crypt has been addressed in many studies. In [1] a threshold is used to identify the gland seeds which are grown to obtain the nuclei chain. In [2] the pixel labelling to different classes is performed using a clustering approach based on the textural properties. In [3] the high level information is preferred against the local one in segmenting the colon glands from hematoxylin-and-eosin stained images. An object-graphs approach is described where the relationship between the primitive objects (nucleus and lumen) is considered. In [4] the prostate cancer malignancy is automatically graded (Gleason system) after the prostate glands are detected.

Many image analysis techniques were successfully used in medical image processing. In [5] the mammographic images are hierarchically decomposed into different resolutions and segmented by analyzing the coarser resolutions while in [6] the multiresolution wavelet analysis is used for texture classification. A Gaussian multiresolution segmentation technique is combined in [7] with the expectation maximization (EM) algorithm to overcome the drawbacks of the EM algorithm. 
The maximally stable extremal region is mostly used in situation where a single threshold is difficult or impossible to select. A general analysis of this segmentation approach is described in [8] and an application in medical images can be found in [9].

\subsection{Aim of the Study}

The basic functional unit of the small intestine is the crypt (crypt of Lieberkühn) [10] and it comprises two main structures of interest: the lumen and the epithelial layer (Fig. 1a). The epithelial layer contains epithelial cells and surrounds the empty area called lumen. The stroma is a heterogeneous compartment between crypts and contains the extra cellular matrix (ECM), fibroblasts, macrophages, vessels etc.

Depending on the marker used on tissues, different components/proteins can be highlighted. To separate the epithelial layer, immunofluorescence staining is performed in paraffin embedded sections with the anti-cytokeratin 8 (CK-8) antibody and a fluorochrome-labelled secondary antibody [11]. The CK-8 is used because it reacts with cytokeratins, proteins found only in the cytoskeleton of epithelial cells. In Fig. 1a, b) the bright area is the binding result between the CK- 8 and the epithelial components; the small dark regions are caused by the epithelial nuclei and their lack of cytokeratins.

This work provides specific techniques (Fig. 2) to automatically segment and measure the crypts (epithelial areas) from fluorescence images of colorectal tissue sections. We used 8 bit greyscale images (Fig. 1a) showing cytokeratins from epithelial layer; they were acquired using the automated TissueFAXS ${ }^{\mathrm{TM}}$ slide scanner (TissueGnostics GmbH, Austria).

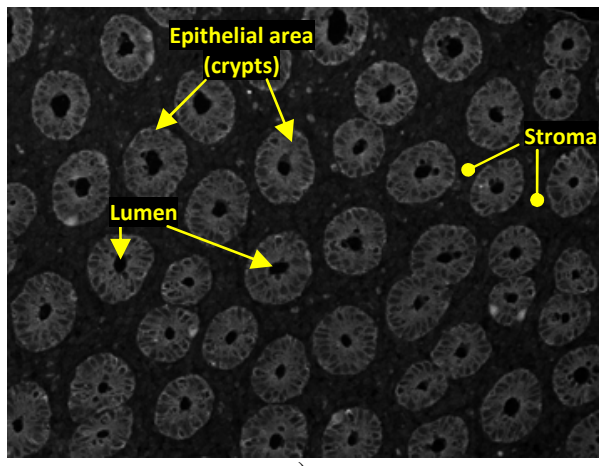

a)

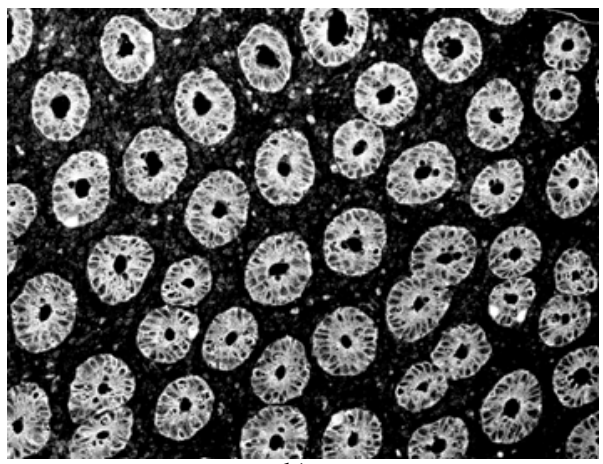

b)

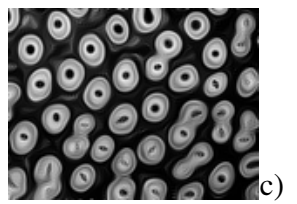

Fig. 1. a) Fluorescence greyscale image showing crypts stained with anti-cytokeratin 8 from colon tissue section. b) The enhanced image by histogram processing; the bright regions indicate the crypts and the inside black regions are the lumens. c) The image from the $4^{\text {th }}$ level of the ADP, up sampled for better visualization. 


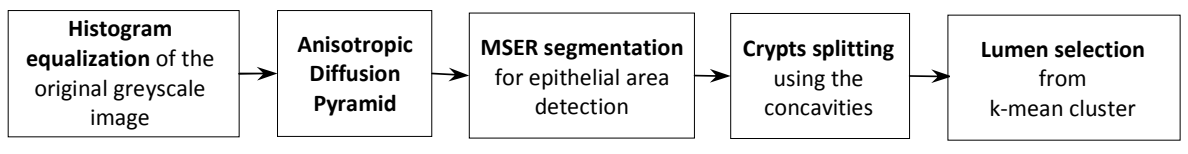

Fig. 2. Overview scheme of the proposed technique

The main motivation for segmenting crypts is to provide the pathologists with quantitative data regarding the epithelial area (crypts boundaries) and epithelium-tostroma ratios. These ratios may provide important information for the assessment of cancer in colon or other organs [12]. Automatic segmentation is much faster than visual analysis and does not suffer from typical human analysis disadvantages like subjectivity and fatigue of the evaluator. Samples from more patients can be evaluated, thus providing data which is more relevant from a statistical point of view.

\section{Image Enhancement}

The CK-8 images are slightly underexposed due to tissue variability and local weak binding of the fluorescence marker. The image contrast can be enhanced such that the separation between the crypts and the stroma is better highlighted. Histogram equalization [13] is used because it maximizes the image contrast by transforming the values in an intensity image so that the intensities are better distributed on the image histogram.

Let $I$ denote the input grey scale image. There are many situations in which some image portions don't contain useful information (Fig. 5a), i.e., they have low intensities due to the lack of CK-8 signal. Only the set of pixels with higher intensities than a certain threshold are considered in the histogram enhancement process, since they are the result of binding between the CK-8 and epithelial cells:

$$
I_{\text {enh }}=\left\{\begin{array}{l}
0, \text { if } I \leq c \cdot t h r_{\mathrm{Otsu}}(I) \\
\text { hist_enh }\left(I \mid I>c \cdot t h r_{\mathrm{Otsu}}(I)\right), \text { else }
\end{array}\right.
$$

where $t h r_{\text {Otsu }}(\cdot)$ computes the threshold for an image using Otsu's method [14], $c$ is fixed to 0.5 in our experiments and the function hist_enh( $\cdot)$ performs the histogram equalization. This technique allows the enhancement of the objects of interest, but keeps the low intensity levels for the background (Fig. 1b).

\section{Crypt Outer Borders Detection}

The borders detection process must ignore the gaps within crypts. The low level cues (pixels, small areas) don't provide enough information to separate the regions having a particular meaning [15]. A way must be found to keep only the important information and to remove the unnecessary details. In order to detect these regions, the role of local information (pixel grey values or gradient) is very important but not 
sufficient; also global information like the region's size and relation with the other region types must be included [15].

In [16] the morphological closing operator is used in a multi-resolution structure; the gaps between nuclei are filled by relating the structure element size to the size of the gaps. This gives good results for images with nuclei, but in our case elements within the stroma near the crypts may be merged by the closing operator. Since an accurate delimitation of the epithelial area is required, the anisotropic filtering is applied to homogenize the crypt regions, fill the inside holes while preserving the (weak) epithelial-stroma edge information.

\subsection{Anisotropic Diffusion Pyramid}

If we use the conventional spatial filters, the edges will be blurred up to losing completely necessary information for crypt assessment. Anisotropic diffusion is a nonlinear filtering method, which improves the image quality significantly while preserving the important boundary information. The image is smoothed by applying a diffusion process whose diffusion tensor acts mainly along the preferred direction [17], [18]. This coherence-enhancing filtering uses the structure tensor of the initial image (in our case the result of the histogram equalization) and a diffusion tensor $D$. This processes is given by an equation of type:

$$
\frac{\partial f}{\partial t}=\nabla \cdot(D \nabla f), \quad D=\left(\begin{array}{ll}
a & b \\
c & d
\end{array}\right)
$$

where $f$ is the evolving image, $t$ denotes the diffusion time and $D$ is the diffusion tensor.

The preferred local orientations are obtained by computing the eigenvectors of the structure tensor ('second-moment matrix') $J_{\rho}$ [18]. The local contrast along these directions is given by the corresponding eigenvalues $\left(\mu_{1}\right.$ and $\left.\mu_{2}\right)$. The diffusion tensor $D$ of the anisotropic diffusion uses the same eigenvectors as the structure tensor $J_{\rho}$ but its eigenvalues $\lambda_{1}, \lambda_{2}$ and the entries of $D$ are computed in as follows [18]:

$$
\lambda_{1}=c_{1}, \lambda_{2}=\left\{\begin{array}{c}
c_{1} \quad \text { if } \mu_{1}=\mu_{2}, \\
c_{1}+\left(1-c_{1}\right) e^{\frac{-c_{2}}{\left(\mu_{1}-\mu_{2}\right)^{2}}} \text { else }
\end{array}, \quad\left[\begin{array}{l}
a=\lambda_{1} \cos ^{2} \alpha+\lambda_{2} \sin ^{2} \alpha, \\
b=\left(\lambda_{1}-\lambda_{2}\right) \sin \alpha \cos \alpha, \\
c=\lambda_{1} \sin ^{2} \alpha+\lambda_{2} \cos ^{2} \alpha
\end{array}\right.\right.
$$

where $0<c_{1}<1$ and $c_{2}>0$.

Let $\zeta^{T}$ denote the anisotropic diffusion process and $T$ the total diffusion time. The anisotropic diffusion pyramid (ADP) [19] $\Pi$ consists of $L$ levels. The first level is the histogram enhance image $\Pi^{1}=I_{\text {enh }}$ (width $w=1392$ and height $h=1024$ ) and each level $\ell>1$ is given by

$$
\Pi^{l}=\zeta^{T}\left(\Pi^{l-1}\right) \downarrow_{4}, \ell=\overline{2, L}
$$


where the $\downarrow_{4}$ denotes the sub-sampling process which reduce the image size by four times (halving the $w$ and $h$, a scale pyramid with one octave between scale).

Using the ADP, the edge sharpening properties of anisotropic diffusion can be exploited together with the performance of the multiresolution hierarchical process. The anisotropic diffusion smooths the objects' interior and removes the dark holes. The gaps are continually filled by going to the coarser levels of the pyramid.

A resolution level must be selected from this coarse-to-fine representation such that the crypts area is accurately delineated and all gaps filled. From experimental tests resulted that the $4^{\text {th }}$ level $(w=174, h=128)$ (critical level) is the maximum level of the pyramid which offers the minimum details to extract relevant information (Fig. 1c and Fig. 5b).

\subsection{MSER for Epithelial Area Detection}

In Fig. 1c and Fig. 5b the bright round areas represent the epithelial regions and the interstitial details were spread out between crypts. Due to the anisotropic diffusion, the pixels intensities in the middle of the "donut" decreased because of the small holes. Although the histogram equalization has been applied for image contrast enhancement, the global or adaptive threshold will not offer stable results.

All thresholds are checked and the connected components are extracted judging their change in area in the maximally stable extremal region (MSER) algorithm [20]. The MSERs are regions that are either darker (MSER-) or brighter (MSER+) than their surroundings and are stable according to a stability criterion across a range of thresholds on the intensity function.

A threshold application on the $4^{\text {th }}$ level of ADP gives the binary image $B_{t}$. The regions in the binary images became smaller by ranging the threshold form 1 to 256 (for 8 bit images). Let $\Psi\left(R_{i}^{t}\right)$ defines the stability based on the area change of region $R_{i}^{t}$ from the image $B_{t}$ obtained with threshold $t$ :

$$
\Psi\left(R_{i}^{t}\right)=\frac{\left|R_{i^{\prime}}^{t-\Delta}\right|-\left|R_{i^{\prime \prime}}^{t+\Delta}\right|}{\left|R_{i}^{t}\right|}
$$

where $\Delta(\Delta=15$ in our experiments $)$ is the stability range parameter and $\mid \cdot$ gives the region area. The regions $R_{i^{\prime}}^{t-\Delta}$ and $R_{i^{\prime \prime}}^{t+\Delta}$ are the ascendant and the descendent regions of $R_{i}^{t}$ obtained by decreasing respectively increasing the threshold level and $R_{i^{\prime}}^{t-\Delta} \supset R_{i}^{t} \supset R_{i^{\prime \prime}}^{t+\Delta}$. A region $R_{i}^{t}$ is MSER+ if it has the following properties:

- $\quad$ extremal, i.e., all pixels inside the connected region are strictly brighter than the surrounding ones.

- stable, i.e., its area changes only slightly with the change of the threshold $t$ (across $2 * \Delta+1$ thresholds).

- $\quad$ maximally stable, i.e., $\Psi\left(R_{i}^{t}\right)$ has a local minimum at $t$. 
The advantage of using the MSER is given by the lack of a global or adaptive threshold. All thresholds are tested and the stability of the connected components evaluated. If multiple stable thresholds exist then a set of nested regions is obtained per object. In this case the output of the MSER detector is not a binary image.

The round dark channel within the 'donut' causes the MSER to produce more than one stable region per crypt, but they are nested. Since we are interested in only one region per crypt, the final binary result is obtained by considering the union of all MSERs.

$$
I_{b w}=\bigcup_{t \in T, i_{t} \in \mathrm{M}_{t}} R_{i_{t}}^{t}
$$

where $T$ is the set of thresholds values for which a MSER has been found and $\mathrm{M}_{t}$ contains the MSERs for the threshold $t$.

This approach has been chosen also for its preference for round regions [8] given by the affine-invariant property [20]. The regions in Fig. 1c and Fig. 5b are homogeneous regions with distinctive boundaries; high detection accuracy especially in these situations has also been concluded in [21].

In case of using the pyramid in image segmentation, the proper reconstruction of the regions found on the top levels must be done by analysing the finer lower levels of the hierarchy where more details are present. In our anisotropic diffusion pyramid this is not required due to the anisotropic filtering which preserves (and even enhance) the boundaries between the epithelial areas and stroma.

The borders of the MSERs+ delimit the epithelial areas and actually represent the outer border of the crypt (the green curves in Fig. 3a). The segmentation produces false positive (FP) results in which two close crypts are detected as a single region (regions A and B in Fig. 3a); the crypts in this situation are rechecked and split in Section 4.1 .

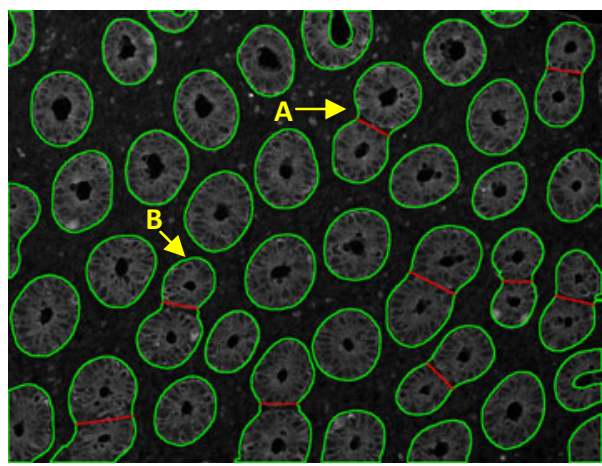

a)

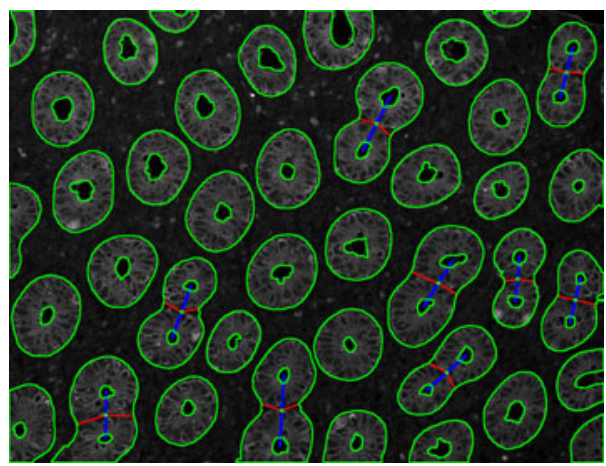

b)

Fig. 3. a) The green curves delimit the epithelial regions and the red lines split the FP results with two close crypts. b) The crypts and the lumens boundaries are depicted in green. In case of two touching crypts the blue line connects the lumens' centroids and the red lines separate the crypts by connecting the two concave points with the middle of the centroids line. 


\section{Crypts Separation and Lumen Detection}

The anisotropic filtration can shrink or even totally fill the lumen; in these cases the MSER- is not able to give good results and MSER+ is unstable. Before performing the lumen detection, the touching crypts segmented as a single region in Section 3.2 are split by considering their geometrical features.

\subsection{Crypts Separation}

The split of the crypts from a FP result (e.g., regions A, B in Fig. 3a) is made by considering the round-like shape, the size and the concavities near the separation area. The FP results are concave regions and the concavities of these regions give critical information about how the crypts can be separated.

For each region $G^{i}$ obtained by MSER+, the convex hull $G_{\text {convex }}^{i}$ can be computed. Considering the round shape of the crypt we expect that $G_{\text {convex }}^{i}=G^{i}$. This is true for the true positive results but for the FP results the difference between the convex hull and the concave region $G_{\text {convex }}^{i}-G^{i} \neq 0$ offers two regions $C_{1}$ and $C_{2}$ on both sides of the crypts (depicted with blue in Fig. 4a). These concavities can be approximated with two triangles and their apexes indicate the entrance into the thin separation area between the two crypts. The separation line is determined by the points $p_{C_{1}} \in C_{1}$ and $p_{C_{2}} \in C_{2}$ such that the distance $d\left(p_{C_{1}}, p_{C_{1}}\right)$ is minimum (pink line in Fig. 4a. This is a rough separation used for detecting the proper lumen per crypt.
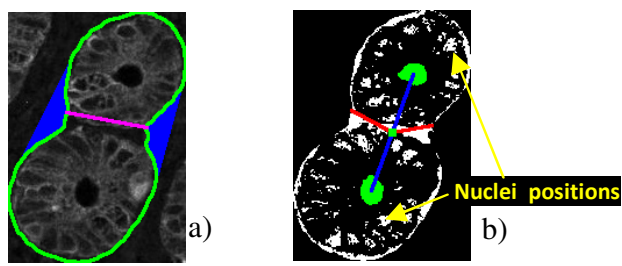

Fig. 4. a) The pink line approximately separates the crypts. b) The cluster with the lowest values contains the lumens (green regions connected by the blue line) and the white regions; the separation line connects the middle of this line with the two concave points.

\subsection{Lumen Detection}

The lack of cytokeratins in the cell nuclei produces the dark regions considerable smaller than the lumen. Three intensity classes exist within a found region (from Section 3.2) corresponding to the background and the noisy pixels (lowest intensities), weak cytokeratins binding and strong cytokeratins binding. These classes are detected using the k-means clustering [13]. The cluster with the lowest values contains the pixels from the lumen and the small dark holes corresponding to the nuclei (Fig. 4b). The proper region $r_{L}$ for lumen is selected by considering its size bigger than the nuclei:

$$
r_{L} \mid \mathrm{A}\left(r_{L}\right)=\max \left\{\mathrm{A}\left(r_{i}, i=\overline{1, N}\right)\right\} \wedge\left(\mathrm{A}\left(r_{L}\right)>a_{\text {min }}\right)
$$


where $\mathrm{A}(\cdot)$ returns de area of a region, $N$ denotes the number of regions from the lowest cluster and $a_{\min }$ is the minimum area of a lumen region. The Fig. $4 \mathrm{~b}$ shows the regions from the lowest cluster (white) and the selected regions as lumen (green).

The set of pixels on which k-means is applied is very important. E.g., in [3], the hematoxylin-and-eosin images are used for glands detection. The pixels are first quantized into a number of clusters and the cluster corresponding to epithelial layer is further processed in the proposed technique. In our case, even though the image was enhanced so that the intensities are better distributed on the image, the k-mean clustering of the entire image doesn't guarantee good results. Instead, the pixels from the small regions found in Section 3.2, which cover one or two crypts, are successfully classified into the proper three clusters.

Even though the exact crypt delineation is not needed (more arguments in Section 5), the separation line can be better approximated by considering the band width of the crypts. By connecting the middle of the centroids line with the concave points found in Section 4.1, a better crypt separation is obtained (Fig. 4b, Fig. 3b). Having better crypt segmentation might enable computing new statistics per crypt once additional markers are used, i.e., percentage of proliferating cells per crypt when staining with a proliferation marker (Ki67).

\section{Results}

The main issue is to segment the epithelial layer by detecting the area covered by crypts; in the case of two touching/very close crypts the outer boundaries detected in 3.2 delimit the epithelial cells. An exact segmentation of the pixels within this area is not really necessary, but the number of crypts must be accurately found since it may be used in computing statistics about crypts distribution within colon tissue. We tested the proposed segmentation technique on different datasets of images from CK-8 labelled tissue sections; some results are show in Fig. 3b, Fig. 5a and Fig. 6.

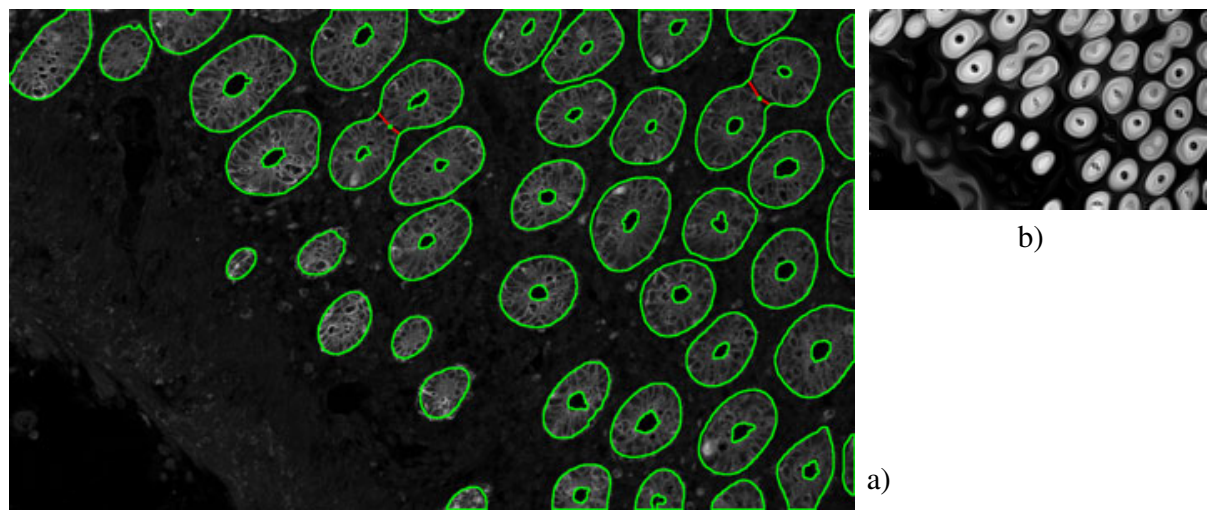

Fig. 5. a) The crypts and the lumens boundaries are depicted in green; the red lines separate the touching crypts. b) The image from the $4^{\text {th }}$ level of the ADP, up sampled for better visualization. 
A more rigorous evaluation must be done by comparing the results against the ground truth segmentations. Since a database with reference segmentations for this type of images does not yet exist, a human expert has been asked to manually create a set of ground truth segmentations (GTS). The performance of the algorithm is established by determining how far the obtained segmentation is from the GTS.

The measures precision $(\mathrm{P})$, recall $(\mathrm{R})$ and accuracy $(\mathrm{A})$ [22] are widely used to characterize the agreement between the region boundaries of two segmentations because they are sensitive to over- and under-segmentation. These measures are defined using the true positive (TP), true negative (TN), FP and false negative (FN).

$$
P=\frac{T P}{T P+F P} ; R=\frac{T P}{T P+F N} ; A=\frac{T P+T N}{T P+T N+F P+F N}
$$

The following results have been obtained by comparing the segmentation of more than 450 crypts: $P=0.952, R=0.944$ and $A=0.947$. The results confirmed that the proposed method could efficiently segment the epithelial area with a high accuracy. Since this evaluation is influenced by the accuracy of the crypt boundaries drawn by the expert, a more suitable approach to properly quantify the performance of the algorithm is still a challenge.
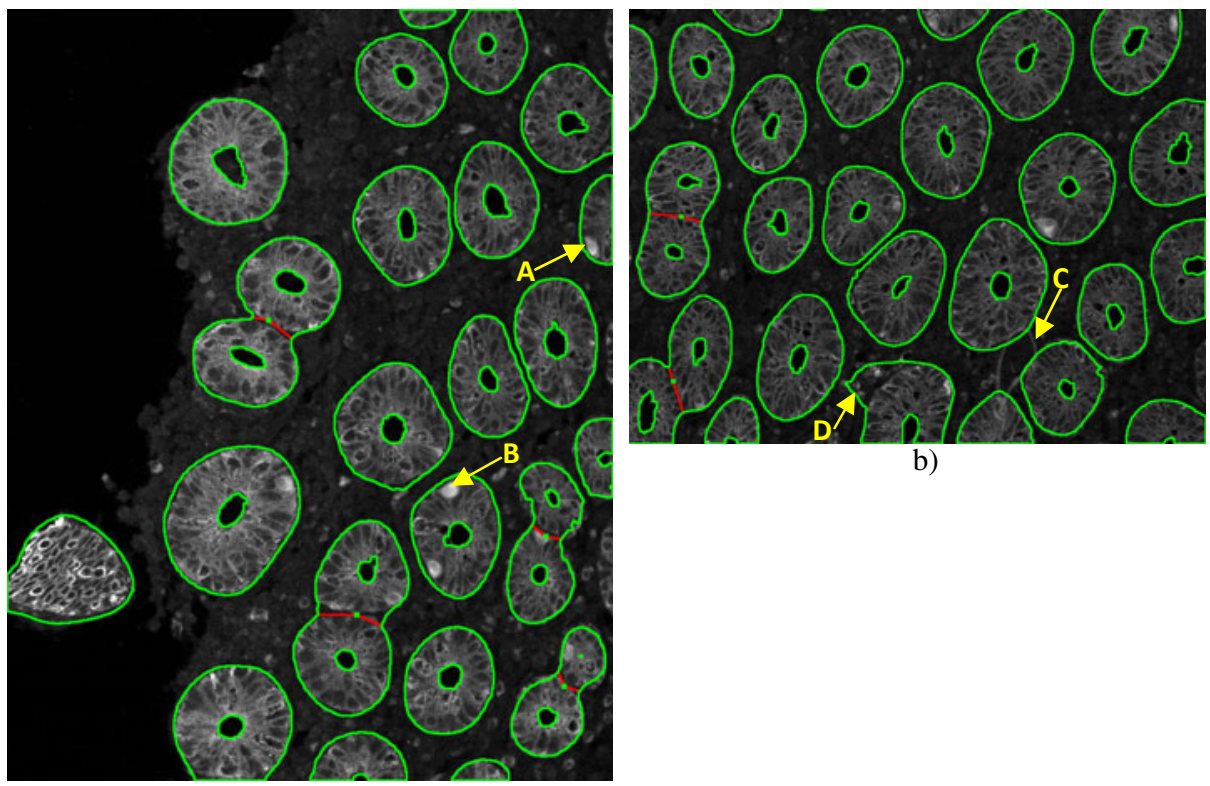

b)

a)

Fig. 6. The crypts and the lumens boundaries are depicted in green; the red lines separate the touching crypts. In a) the bright spots (regions A and B) were correctly included in the crypt region. In b) region $\mathrm{C}$ indicates an artefact overlapping the crypts but eliminated due to ADP. Region D is a case FP segmentation. 


\section{Conclusions}

A new automatic technique for robust crypt segmentation based on anisotropic pyramid and stable regions is presented in this paper. The ADP is used to highlight the crypt positions and the MSER algorithm segments the critical level where only the important information persisted. The k-means clustering is used to identify the lumens and the geometrical information is used to split the touching crypts.

The developed segmentation method allows automated assessment of crypts in microscopic images from colorectal tissue sections. Areas of epithelium and stroma are properly identified across a statistically relevant image dataset. The developed technique can be used to assess the areas of epithelium and stroma and their ratios in large tissues sections. The information provided by these measurements allows a better understanding of epithelium-stroma interactions in colon tissue, as well as to confirm the existing qualitative knowledge with new quantitative measurements.

This technique uses a coarser-to-fine approach and can be easily extended on any image with epithelial area from different tissues types (e.g., prostate, breast or lung) but also in any other field in which the objects of interest have the features considered in designing this method. This study will be continued by analysing the topological properties of the graph associated to the tissues components. Considerable effort will be spent to obtain a database with ground-truth segmentations and to find rigorous evaluation criteria of the results.

Acknowledgments. This work was supported in part by the "BRAIN - An Investment in Intelligence" doctoral Scholarship Program within the Technical University of Iasi, Romania, by the Austrian Science Fund under grant no. P20134-N13 and by FFG BRIDGE 818094. The images and necessary biomedical explanations were kindly provided by Dr. Giovanna Bises from the Institute of Pathophysiology and Allergy Research, Medical University of Vienna.

\section{References}

1. Wu, H.S., Xu, R., Harpaz, N., Burstein, D., Gil, J.: Segmentation of intestinal gland images with iterative region growing. Journal of Microscopy 220(3), 190-204 (2005)

2. Farjam, R., Soltanian-Zadeh, H., Jafari-Khouzani, K., Zoroofi, R.A.: An image analysis approach for automatic malignancy determination of prostate pathological images. Clinical Cytometry 72B(4), 227-240 (2007)

3. Gunduz-Demir, C., Kandemir, M., Tosun, A.B., Sokmensuer, C.: Automatic segmentation of colon glands using object-graphs. Medical Image Analysis 14(1), 1-12 (2010)

4. Naik, S., Doyle, S., Feldman, M., Tomaszewski, J., Madabhushi, A.: Gland segmentation and computerized Gleason grading of prostate histology by integrating low-, high-level and domain specific information. In: 2nd MICCAI Workshop on Microscopic Image Analysis with Application in Biology, Piscataway, NJ, USA (2007)

5. Chen, C.H., Lee, G.G.: Image Segmentation Using Multiresolution Wavelet Analysis and Expectation-Maximization (EM) Algorithm for Digital Mammography. International Journal of Imaging Systems and Technology 8(5), 491-504 (1997) 
6. Roshni, V.S., Raju, G.: Image Segmentation using Multiresolution Texture Gradient and Watershed Algorithm. International Journal of Computer Applications 22(5), 21-28 (2011)

7. Tolba, M.F., Mostafa, M.G., Gharib, T.F., Salem, M.A.: MR-Brain Image Segmentation Using Gaussian Multiresolution Analysis and the EM Algorithm. In: Proc. ICEIS (2), pp. $165-170$ (2003)

8. Kimmel, R., Zhang, C., Bronstein, A., Bronstein, M.: Are MSER features really interesting?. IEEE Trans. PAMI (2010) (in press)

9. Donoser, M., Bischof, H.: 3D Segmentation by Maximally Stable Volumes (MSVs). In: 18th International Conference on Pattern Recognition (ICPR 2006), vol. 1, pp. 63-66 (2006)

10. Humphries, A., Wright, N.A.: Colonic Crypt Organization and Tumorigenesis: Human Colonic Crypts. Nature Reviews Cancer 8(6), 415-424 (2008)

11. Moll, R., Divo, M., Langbein, L.: The human keratins: biology and pathology. Histochemistry and Cell Biology 129(6), 705-733 (2008)

12. de Kruijf, E.M., van Nes, J.G.H., van de Velde, C.J.H., Putter, H., Smit Vincent, T.H.B.M., Liefers Gerrit, J., Kuppen, P.J.K., Tollenaar, R.A.E.M., Mesker, W.E.: Tumorstroma ratio in the primary tumor is a prognostic factor in early breast cancer patients, especially in triple-negative carcinoma patients. Breast Cancer Research and Treatment 125(3), 687-696 (2011)

13. Gonzalez, R.C., Woods, R.E.: Digital Image Processing, 3rd edn. Addison-Wesley, Reading (1992)

14. Otsu, N.: A Threshold Selection Method from Gray-Level Histograms. IEEE Transactions on Systems, Man, and Cybernetics 9(1), 62-66 (1979)

15. Kropatsch, W.G., Haxhimusa, Y., Ion, A.: Multiresolution Image Segmentations in Graph Pyramids. Applied Graph Theory in Computer Vision and Pattern Recognition 52, 3-41 (2007)

16. Smochina, C., Manta, V., Kropatsch, W.G.: Semantic Segmentation of Microscopic Images using a Morphological Hierarchy. In: Real, P., Diaz-Pernil, D., Molina-Abril, H., Berciano, A., Kropatsch, W. (eds.) CAIP 2011, Part I. LNCS, vol. 6854, pp. 102-109. Springer, Heidelberg (2011)

17. Weickert, J.: Anisotropic Diffusion in Image Processing. Teubner, Stuttgart (1998)

18. Scharr, H., Weickert, J.: An anisotropic diffusion algorithm with optimized rotation invariance. In: German Association for Pattern Recognition, Kiel, Germany, pp. 460-467 (September 2000)

19. Acton, S.T., Bovik, A.C., Crawford, M.M.: Anisotropic diffusion pyramids for image segmentation. In: Proceedings of 1st IEEE International Conference on Image Processing (ICIP 1994), vol. 3, pp. 478-482 (1994)

20. Matas, J., Chum, O., Urban, M., Pajdla, T.: Robust wide baseline stereo from maximally stable extremal regions. In: Proc. BMVC, pp. 384-393 (2002)

21. Mikolajczyk, K., Tuytelaars, T., Schmid, C., Zisserman, A., Matas, J., Schaffalitzky, F., Kadir, T., Van Gool, L.: A comparison of affine region detectors. International Journal of Computer Vision 65, 43-72 (2005)

22. Chalana, V., Kim, Y.: A methodology for evaluation of boundary detection algorithms on medical images. IEEE Trans. Med. Imag. 16(5), 642-652 (1997) 